\title{
Stability of nonlinear discrete repetitive processes with Markovian switching
}

\author{
Julia Emelianova a , Pavel Pakshin ${ }^{\mathrm{a}, \mathrm{b}, *}$, Krzysztof Gałkowski $^{\mathrm{c}}$, Eric Rogers ${ }^{\mathrm{d}}$ \\ ${ }^{a}$ Arzamas Polytechnic Institute of R.E. Alekseev Nizhny Novgorod State Technical University, 19, Kalinina Street, Arzamas, 607227, Russia \\ ${ }^{\mathrm{b}}$ Lobachevsky State University of Nizhny Novgorod, Prospekt Gagarina, 23, 603950, Nizhny Novgorod, Russia \\ ${ }^{\mathrm{c}}$ Institute of Control and Computation Engineering, University of Zielona Góra, ul. Podgórna 50, 65-246 Zielona Góra, Poland \\ ${ }^{\mathrm{d}}$ Electronics and Computer Science, University of Southampton, Southampton SO17 1BJ, UK
}

\section{A R T I C L E I N F O}

\section{Article history:}

Received 19 September 2013

Received in revised form

23 September 2014

Accepted 2 October 2014

Available online 15 November 2014

Keywords:

Nonlinear repetitive processes

Markovian switching

Stability

Robust control

Iterative learning control

\begin{abstract}
A B S T R A C T
Repetitive processes are a class of 2D systems that operate over a subset of the upper-right quadrant of the 2D plane. Applications include iterative learning control where experimental verification has been reported based on a linear time-invariant model approximation of the dynamics. This paper considers discrete nonlinear repetitive processes with Markovian switching and applies, as one application, the resulting stability theory to iterative learning control for a class of networked systems where time-varying dynamics arise.
\end{abstract}

(C) 2014 Elsevier B.V. All rights reserved.

\section{Introduction}

The systems considered in this paper repeat the same finite duration operation over and over again. Each repetition is termed a pass and the duration the pass length. One industrial application is long-wall coal mining where the coal is cut by a machine that passes along the coal face and the objective is to maximize the volume of coal cut without penetrating the coal/stone interface. During each pass the machine rests on the pass profile cut during the previous pass, i.e., the height of the stone/coal interface above some datum line. Once a pass is complete, the machine is returned to the starting location and then pushed across to rest on the newly cut floor profile ready for the start of the next pass.

The geometry of the long-wall coal mining process means that the previous pass profile acts as a forcing function on the next

\footnotetext{
This work was performed in framework of Contract No 2.1748.2014/K with the Ministry of Education and Science of Russia and also supported in part by the Russian Foundation for Basic Research under grant 13 - 08 - 01092_a, and the National Science Center in Poland under grant 2011/01/B/ST17/00475; Section 3 was supported solely by the Russian Science foundation under grant RSF 14-2900142.

* Corresponding author at: Arzamas Polytechnic Institute of R.E. Alekseev Nizhny Novgorod State Technical University, 19, Kalinina Street, Arzamas, 607227, Russia.

E-mail address: pakshin@apingtu.edu.ru (P. Pakshin).
}

pass profile and hence contributes to its dynamics. This interaction between successive pass profiles can result in oscillations that increase in amplitude from pass-to-pass. These oscillations are the distinguishing feature of repetitive processes [1] and they cannot be removed by standard control action. Instead these processes must be treated as systems operating over a subset of the upperright quadrant of the $2 \mathrm{D}$ plane.

This paper uses the notation $y_{k}(t), 0 \leq t \leq T$, where $y$ is the scalar or vector valued variable, $k \geq 0$, is the pass number and $T<\infty$ is the pass length. Given that these process operate over the domain defined by $(k, t) \in[0, \infty] \times[0, T]$, boundary conditions need to be specified for $k=0$ and $t=0$, i.e, the starting, or initial, condition on each pass and the initial pass profile respectively. The detailed modeling of long-wall coal mining as a repetitive process is given in [1], which also references the original work and details the modeling of other repetitive processes, such as forms of metal rolling.

In physical examples, such as long-wall coal mining, the interaction between success pass profiles is part of the evolution of the dynamics. Of direct relevance to the focus of this paper are applications where the repetitive process structure arises from the control action applied. Consider the commonly encountered industrial task where a gantry robot is undertaking a pick and place operation over and over again and the sequence of operations is: (i) collect an object from a specified location, (ii) transfer it over a 
finite duration, (iii) deposit it at a fixed location or onto a moving conveyor, (iv) return to the starting location, and (v) repeat (i)-(iv) as many times as required or until a stop for maintenance is required. The transfer of each object can be viewed as completing a pass over the finite pass length and once complete, all information generated is available to update the control law to be applied on the next pass.

Iterative Learning Control (ILC), see the survey papers [2,3], has been developed for applications such as the gantry robot operation outlined briefly above. The control law is usually computed in the resetting time between successive passes as a function of the control used on the previous pass and a corrective term computed using information from the previous pass (also termed a trial in some literature) input and error or a finite number thereof. Suppose that a reference signal, $y_{\text {ref }}(t)$ that can be realized by the gantry robot is given and on pass $k$ let $y_{k}(t), 0 \leq t \leq T$, be the pass profile and $u_{k}(t)$ the control input. Then $e_{k}(t)=$ $y_{\text {ref }}(t)-y_{k}(t)$ is the error on this pass and the ILC design problem to force tracking of the reference can be formulated as control law design to achieve convergence, as measured by the norm on the underlying function space, to zero of the error sequence $\left\{e_{k}\right\}_{k}$ and convergence of the input sequence $\left\{u_{k}\right\}_{k}$ to $u_{\infty}$, which is termed the learned control. Another example where the repetitive process structure arises as a result of control action or the iterative solution method is nonlinear dynamic optimal control problems based on the maximum principle (described in [1] with references to the original work).

Given the possibility of oscillations that increase in amplitude from pass-to-pass, stability of a repetitive process is defined as requiring that a bounded initial pass profile produces a bounded sequence of pass profiles, defined in terms of the norm on the underlying function space, either over the finite pass length or for all possible values of this parameter. If the dynamics are linear then an abstract model in a Banach space setting can be used [1] where the conditions are expressed in terms of the bounded linear operator describing the contribution of the previous pass profile to the dynamics of the next. This theory has been applied to ILC design, assuming that the dynamics are time-invariant, with experimental verification [4] on a gantry robot that replicates the pick and place operation discussed briefly above.

A significant proportion of the literature on the control of $2 \mathrm{D}$ systems is based on a linear model of the dynamics. Comparatively little work has been reported on the stability of nonlinear or linear time-varying examples, see, for example, [5]. In many possible applications for repetitive processes/2D systems, the dynamics are nonlinear and the new results in this paper address this issue with an application to ILC where it is shown that control law design by Linear Matrix Inequalities (LMIs) is possible for cases where linearization of the nonlinear dynamics about an operating point is possible. One more recent addition to applications for repetitive process control theory where the use of a nonlinear model will be required is laser metal deposition processes [6]

In the application of control systems, failures in operation can arise and the representation used in this paper for this problem is based on random switching. In particular, a process with failures is modeled by a state-space model with jumps in the parameter values and/or structure governed by a Markov chain with a finite set of states, often termed Markovian jump systems or systems with random structure, see, for example, [7]. Results on the development of control theory for Markovian jump systems, which address issues such as stability, optimal and robust control problems, in the standard, or 1D, case can be found in, for example, [8-13]. These results cannot be applied to 2D systems. Progress on the development of a systems theory for 2D linear systems with Markovian jumps is reported in $[14,15]$ and references therein.

This paper considers nonlinear and time-varying discrete repetitive processes where, with applications such as ILC over a network in mind, the dynamics also have Markovian switching. The property of exponential stability in the mean square is defined and characterized, leading to results on stabilization and $\mathscr{H}_{\infty}$ control with an application to ILC. Moreover, the results are developed for time-invariant dynamics but have an immediate extension to the time-varying case. As in other control systems areas, it is to be expected that progress towards control law design will for physical examples with nonlinear dynamics make use of particular features in the corresponding models. Exactly this feature is present in the ILC design analysis that forms the second major part of this paper.

\section{Process description and stability theory}

If the dynamics of a repetitive process are linear then stability analysis can proceed from the abstract model and the task for a given example is to obtain conditions that can be tested. Let the pass profile $y_{k} \in E_{T}$ where $E_{T}$ is a Banach space. Then the passto-pass dynamics of a linear repetitive process with constant pass length $T<\infty$ are described by $y_{k+1}=L_{T} y_{k}, k \geq 0$, where $L_{T}$ is a bounded linear operator mapping $E_{T}$ into itself. In this case $L_{T}$ is a convolution operator over the finite interval $t \in[0, T]$ and contributions that enter on the current pass can be represented by adding a term that lies in a linear subspace of $E_{T}$.

The stability problem for repetitive processes is that the pass profile sequence $\left\{y_{k}\right\}_{k \geq 1}$ for a given initial profile $y_{0}$ can contain oscillations that increase in amplitude with $k$, as discussed in the previous section for the coal cutting example. Bounded-Input Bounded-Output (BIBO) stability for these processes is therefore defined, in terms of the norm on the underlying function space, as the requirement that a bounded initial pass profile produces a bounded sequence $\left\{y_{k}\right\}_{k \geq 1}$, either over the finite pass length or else independent of this parameter. The latter property is the most general case and for dynamics described by the abstract model, i.e., by $L_{T}$, requires the existence of real numbers $M_{\infty}>0$ and $\lambda_{\infty} \in(0,1)$, which are independent of $T$, such that $\left\|L_{T}^{k}\right\| \leq M_{\infty} \lambda_{\infty}^{k}$ where $\|\cdot\|$ denotes both the norm on $E_{T}$ and the induced operator norm.

The vast majority of the systems theory currently available for repetitive processes is for linear deterministic examples or those for which such a description is an adequate basis for initial analysis. In this paper, the process state-space model considered is

$$
\begin{aligned}
& x_{k+1}(t+1)=F_{1}\left(x_{k+1}(t), y_{k}(t), u_{k+1}(t), w_{k}(t), r_{k}(t)\right), \\
& y_{k+1}(t)=F_{2}\left(x_{k+1}(t), y_{k}(t), u_{k+1}(t), w_{k}(t), r_{k}(t)\right), \\
& 0 \leq t \leq T, k=0,1,2, \ldots
\end{aligned}
$$

where the integer $T$ denotes the pass length and on pass $k, x_{k}(t) \in$ $\mathbb{R}^{n_{x}}$ is the current pass state vector, $y_{k}(t) \in \mathbb{R}^{n_{y}}$ is the pass profile vector, $u_{k}(t) \in \mathbb{R}^{n_{u}}$ is the control input vector, $w_{k}(t) \in \mathbb{R}^{n_{w}}$ is the disturbance vector, $F_{1}$ and $F_{2}$ are nonlinear functions, $r(t)$ is the homogeneous Markov chain whose state-space is the set of integers $\mathbb{N}=\{1,2, \ldots, v\}$ and the transition probabilities are given by

$\mathrm{P}\left[r_{k}(t+1)=j \mid r_{k}(t)=i\right]=\pi_{i j}$,

$\mathrm{P}\left[r_{k+1}(t)=j \mid r_{k}(t)=i\right]=\omega_{i j}$.

The boundary conditions are the pass state initial vector sequence and the initial pass profile and in this paper have the form

$x_{k+1}(0)=d_{k+1}, \quad k \geq 0$,

$y_{0}(t)=f(t), \quad 0 \leq t \leq T-1$,

where the entries in the $n \times 1$ vector $d_{k+1}$ are known constants and $f(t)$ is an $m \times 1$ vector whose entries are known functions of $t$. Also the presence of the time shift on the left-hand side of the first equation in (1) means that the state vector $x_{k}(t)$ is defined over 
$0 \leq t \leq T$, including the specified initial state vector $(t=0)$ and the remaining vectors over $0 \leq t \leq T-1$.

Let $|q|$ denote the Euclidian norm on the vector $q$ and $\mathrm{E}$ the expectation operator. Suppose also that there exists a finite real number $M_{f}>0$ such that $d_{k+1}$ and $f(t)$ satisfy

$\left|d_{k+1}\right|^{2} \leq \kappa_{d} \eta_{d}^{k+1}, \quad \kappa_{d}>0,0<\eta_{d}<1, k=0,1, \ldots$

$|f(t)| \leq M_{f}, \quad 0 \leq t \leq T-1$.

Except where stated otherwise, all future references to the boundary conditions in this paper will assume that they satisfy (3) and it is also assumed that for each $r \in \mathbb{N}$

$F_{1}(0,0,0,0, r)=0, \quad F_{2}(0,0,0,0, r)=0$.

The following is the definition of stability for the repetitive processes considered in this paper.

Definition 1. A discrete nonlinear repetitive process described by (1) and (2) is said to be exponentially stable in the mean square, or exponentially stable for short, if

$\mathrm{E}\left[\left|x_{k+1}(t)\right|^{2}+\left|y_{k}(t)\right|^{2}\right] \leq \kappa \eta^{k+t}, \quad \kappa>0,0<\eta<1$.

To study stability, a vector Lyapunov function approach is used where it is convenient to define from (1)

$\bar{x}_{k}(t)=\left[x_{k+1}^{T}(t) y_{k}^{T}(t)\right]^{T}$.

The candidate function is

$V\left(\bar{x}_{k}(t), r_{k}(t)\right)=\left[\begin{array}{c}V_{1}\left(x_{k+1}(t), r_{k}(t)\right) \\ V_{2}\left(y_{k}(t), r_{k}(t)\right)\end{array}\right]$,

with $V_{1}(0, r)=0, V_{2}(0, r)=0$. Define the operator $\mathscr{D}$ along the trajectories of (1) as the stochastic discrete counterpart of divergence

$$
\begin{aligned}
& D_{\bar{x}, u, w, r} V\left(\bar{x}_{k}(t), r_{k}(t)\right)=\mathrm{E}\left[V_{1}\left(x_{k+1}(t+1), r_{k}(t+1)\right)\right. \\
& \quad+V_{2}\left(y_{k+1}(t), r_{k+1}(t)\right)-V_{1}\left(x_{k+1}(t), r_{k}(t)\right) \\
& \quad-V_{2}\left(y_{k}(t), r_{k}(t)\right) \mid x_{k+1}(t)=x, \\
& \left.y_{k}(t)=y, u_{k}(t)=u, w_{k}(t)=w, r_{k}(t)=r\right]
\end{aligned}
$$

and the following result can be established.

Theorem 1. Consider a discrete nonlinear repetitive process described by (1) and (2). Suppose also that there exist positive constants $c_{1}, c_{2}, c_{3}$, with $c_{2}>c_{3}$, such that $V$ and $D$ satisfy

$c_{1}|x|^{2} \leq V_{1}(x, r) \leq c_{2}|x|^{2}$,

$c_{1}|y|^{2} \leq V_{2}(y, r) \leq c_{2}|y|^{2}$,

$\mathscr{D}_{\bar{x}, 0,0, r} V\left(\bar{x}_{k}(t), r_{k}(t)\right) \leq-c_{3}\left(|x|^{2}+|y|^{2}\right), \quad r \in \mathbb{N}$.

Then exponential stability holds.

Proof. Using (8)-(10)

$\mathrm{E}\left[V_{1}\left(x_{k+1}(t+1), r_{k}(t+1)\right)+V_{2}\left(y_{k+1}(t), r_{k+1}(t)\right)\right]$

$$
\leq \lambda \mathrm{E}\left[V_{1}\left(x_{k+1}(t), r_{k}(t)\right)+V_{2}\left(y_{k}(t), r_{k}(t)\right)\right],
$$

where $\lambda=\frac{c_{2}-c_{3}}{c_{2}} \in(0,1)$ by construction. Evaluating (11) in $k$ and $t$ from 0 to $N$ under the condition $k+t=N$, summing the resulting inequalities, adding $V_{1}\left(x_{N+2}(0)\right)+V_{2}\left(y_{0}(N+1)\right)$ to both sides of the result and rearranging gives

$$
\begin{aligned}
\sum_{n=0}^{N+1} \mathrm{E}\left[V_{1}\left(x_{N+2-n}(n), r_{N+1-n}(n)\right)+V_{2}\left(y_{N+1-n}(n), r_{N+1-n}(n)\right)\right] \\
\leq \lambda \sum_{n=0}^{N} \mathrm{E}\left[V_{1}\left(x_{N+1-n}(n), r_{N-n}(n)\right)+V_{2}\left(y_{N-n}(n), r_{N-n}(n)\right)\right] \\
\quad+\mathrm{E}\left[V_{1}\left(x_{N+2}(0), r_{N+1}(0)\right)+V_{2}\left(y_{0}(N+1), r_{0}(N+1)\right)\right] .
\end{aligned}
$$

Considering this last inequality in terms of

$W(N)=\sum_{n=0}^{N} E\left[V_{1}\left(x_{N+1-n}(n), r_{N-n}(n)\right)+V_{2}\left(y_{N-n}(n), r_{N-n}(n)\right)\right]$

gives

$$
\begin{gathered}
\sum_{n=0}^{N} \mathrm{E}\left[V_{1}\left(x_{N+1-n}(n), r_{N-n}(n)\right)+V_{2}\left(y_{N-n}(n), r_{N-n}(n)\right)\right] \\
\leq \sum_{n=0}^{N} \lambda^{N-n} \mathrm{E}\left[V_{1}\left(x_{n+1}(0), r_{n}(0)\right)+V_{2}\left(y_{0}(n), r_{0}(n)\right)\right] .
\end{gathered}
$$

Also for the specified boundary conditions

$\mathrm{E}\left[\left|x_{N+1-t}(t)\right|^{2}+\left|y_{N-t}(t)\right|^{2}\right] \leq \frac{c_{2}}{c_{1}}\left[\frac{\kappa_{d}}{1-\bar{\eta}}+\sum_{t=0}^{T-1} \lambda^{-t}|f(t)|^{2}\right] \tilde{\eta}^{N}$,

where $\tilde{\eta}=\max \{\bar{\eta}, \lambda\}, \bar{\eta}=\eta_{*}^{\frac{1}{2}}, \eta_{*}=\max \left\{\eta_{d}, \lambda\right\}$. Consequently (4) holds with $\kappa=\frac{c_{2}}{c_{1}}\left[\frac{\kappa_{d}}{1-\bar{\eta}}+\sum_{t=0}^{T-1} \lambda^{-t}|f(t)|^{2}\right], \quad \eta=\tilde{\eta}$ and the proof is complete.

The following corollary to this last result holds for boundary conditions that also satisfy

$\sum_{k=0}^{\infty}\left|d_{k+1}\right|^{2}+\sum_{t=0}^{T-1}|f(t)|^{2}<\infty$

Corollary 1. Consider a discrete nonlinear repetitive process described by (1) and boundary conditions satisfying (13). Suppose that there exist positive constants $c_{1}, c_{2}, c_{3}$, with $c_{2}>c_{3}$, such that $V$ and $\mathscr{D}$ of (6) and (7), respectively, satisfy (8)-(10). Then

$\mathrm{E}\left[\left|x_{k+1}(t)\right|^{2}+\left|y_{k}(t)\right|^{2}\right] \rightarrow 0$

as $k+t \rightarrow \infty$.

Proof. Summing inequalities (12) in the variable $N$ gives

$$
\begin{aligned}
\sum_{N=0}^{M} W(N) \leq & c_{2} \sum_{k=0}^{M} \lambda^{k}\left(\left|d_{1}\right|^{2}+|f(0)|^{2}\right) \\
& +c_{2} \sum_{k=0}^{M-1} \lambda^{k}\left(\left|d_{2}\right|^{2}+|f(1)|^{2}\right)+\cdots+c_{2} \lambda \\
& \times\left(\left|d_{M}\right|^{2}+|f(M-1)|^{2}\right)+c_{2}\left(\left|d_{M+1}\right|^{2}+|f(M)|^{2}\right) \\
\leq & c_{2} \sum_{k=0}^{\infty} \lambda^{k}\left[\sum_{N=0}^{M}\left(\left|d_{N+1}\right|^{2}+|f(N)|^{2}\right)\right] \\
= & \frac{c_{2}}{1-\lambda} \sum_{N=0}^{M}\left(\left|d_{N+1}\right|^{2}+|f(N)|^{2}\right) .
\end{aligned}
$$

The right-hand side of the last inequality is bounded as $M \rightarrow \infty$ since $0<\lambda<1$ and (13) holds. Hence the series on the left-hand side is convergent and $W(N) \rightarrow 0$ as $N \rightarrow \infty$. Given (8) and (9), it follows that

$\sum_{n=0}^{N} \mathrm{E}\left[\left|x_{N+1-n}(n)\right|^{2}+\left|y_{N-n}(n)\right|^{2}\right] \leq c_{1}^{-1} W(N) \rightarrow 0$,

as $N \rightarrow \infty$. Consequently (14) holds and the proof is complete. 
It follows from the proof of Theorem 1 that this result is also valid for more general time-varying discrete nonlinear repetitive processes described by

$$
\begin{gathered}
x_{k+1}(t+1)=\bar{F}_{1}\left(x_{k+1}(t), y_{k}(t), u_{k}(t), w_{k}(t), r_{k}(t), t\right), \\
y_{k+1}(t)=\bar{F}_{2}\left(x_{k+1}(t), y_{k}(t), u_{k}(t), w_{k}(t), r_{k}(t), t\right), \\
0 \leq t \leq T, k=0,1,2, \ldots
\end{gathered}
$$

where $\bar{F}_{1}$ and $\bar{F}_{2}$ are nonlinear functions such that

$\bar{F}_{1}(0,0,0,0, r, t)=0$,

$\bar{F}_{2}(0,0,0,0, r, t)=0$

and the remaining notation is the same as for (1).

Given this general stability theory for nonlinear processes, research on the structure and design of control laws can begin. In some applications, disturbance attenuation will also arise. As with other nonlinear control systems problems, the aim will be to develop general results but cases of applications interest could arise where the dynamics have particular structural properties that can be exploited to advantage, i.e. akin to electro-mechanical systems. In this context, the next section develops a solution to the stabilization and disturbance attenuation problem for the general model and then in Section 5 application to ILC design for an applications relevant sub-class is considered. The result is LMI based stability tests and control law design algorithms that allows for linear time-varying dynamics as an approximation about an operating point of nonlinear dynamics, whereas the current experimentally verified repetitive process based ILC [4] designs require time-invariant models.

\section{Stabilization and $\mathscr{H}_{\infty}$ disturbance attenuation}

The problem considered in this section is stabilization and attenuation of the effects of the disturbance term $w$ in (1) on, in general, some of the entries in the pass profile vector as defined by the auxiliary measurement

$z_{k}(t)=G\left(x_{k}(t), y_{k}(t), u_{k}(t), w_{k}(t), r_{k}(t)\right)$,

where $G$ is a nonlinear function such that $G(0,0,0,0, r)=0$, in terms of an $\mathscr{H}_{\infty}$ norm. In particular, consider $w_{k}(t) \in l_{2}([0, \infty)$, $[0, \infty)$ ) and introduce

$\|w\|_{2}=\sqrt{\sum_{k=0}^{\infty} \sum_{t=0}^{\infty}\left|w_{k}(t)\right|^{2}}<\infty$.

Define also the norm on $z_{k}(t)$ as

$\|z\|_{\mathrm{E}}=\sqrt{\mathrm{E}\left[\sum_{k=0}^{\infty} \sum_{t=0}^{\infty}\left|z_{k}(t)\right|^{2}\right] .}$

Disturbance attenuation as measured by an $\mathscr{H}_{\infty}$ norm for the processes considered in this paper is defined as follows where no loss of generality arises from assuming zero boundary conditions for the remaining part of this section and hence the formal statements of the new results are given without explicit reference to these conditions.

Definition 2. A discrete nonlinear repetitive process described by (1) and (16) is said to be exponentially stable with prescribed $\mathscr{H}_{\infty}$ disturbance attenuation level $\gamma$ if it is exponentially stable and for zero boundary conditions and for all $w_{k}(t) \in l_{2}([0, \infty)$, $[0, \infty)) \neq 0$

$\|z\|_{\mathrm{E}}<\gamma\|w\|_{2}$.
Recall the definition of the vector $\bar{x}_{k}(t)$ in (5) in the previous section and write $u \in \Phi$, if $u=\varphi(\bar{x}, r)$, where $\varphi$ is a nonlinear function such that $\varphi(0, r)=0, r \in \mathbb{N}$. This control law is activated by the current pass state and previous pass profile vectors. It hence has a feedback/feedforward structure, i.e., feedback of the current state vector and feedforward of the previous pass vector.

Suppose also that $L(\bar{x}, u, r)$ is a nonlinear function such that $L(\bar{x}, u, r) \geq c\left(|\bar{x}|^{2}+|u|^{2}\right)$. Then the following result for the disturbance free case is established by following the steps of the proof of Theorem 1 with routine changes and is hence omitted.

Theorem 2. Assume that for some $u=\varphi(\bar{x}, r) \in \Phi$ the inequality

$\mathscr{D}_{\bar{x}, \varphi, 0, r} V\left(\bar{x}_{k}(t), r_{k}(t)\right)+L\left(\bar{x}_{k}(t), \varphi\left(\bar{x}_{k}(t), r_{k}(t)\right)\right) \leq 0$

has a solution $V\left(\bar{x}_{k}(t), r_{k}(t)\right)$ satisfying (8) and (9). Then a discrete nonlinear disturbance free repetitive process described by (1) and (16) with $u_{k}(t)=\varphi\left(\bar{x}_{k}(t), r_{k}(t)\right)$ is exponentially stable.

The following result solves the disturbance attenuation problem of this section.

Theorem 3. Assume that for some $u=\varphi(\bar{x}, r) \in \Phi$ the inequality

$\mathscr{D}_{\bar{x}, \varphi, w, r} V\left(\bar{x}_{k}(t), r_{k}(t)\right)+\varepsilon|\bar{x}|^{2}+|z|^{2}-\gamma^{2}|w|^{2} \leq 0$

has a solution $V(\bar{x}, r)$, satisfying (8) and (9). Then the discrete nonlinear repetitive process obtained by applying $u=\varphi(\bar{x}, r)$ to (1) and (16) is exponentially stable with prescribed $\mathscr{H}_{\infty}$ disturbance attenuation level $\gamma$.

Proof. Let the pair $(V(\bar{x}), \varphi(\bar{x}, r))$ be a solution of (17) and if $w_{k}(t) \equiv 0$ then

$\mathscr{D}_{\bar{x}, \varphi, 0, r} V\left(\bar{x}_{k}(t), r_{k}(t)\right)+\varepsilon|\bar{x}|^{2} \leq 0$

and since (8), (9) and (18) hold the controlled process is exponentially stable by Theorem 2 .

Setting $w_{k}(t) \in l_{2}([0, \infty),[0, \infty)) \neq 0$, assuming zero boundary conditions and using (17) gives

$$
\begin{aligned}
\mathrm{E}[ & \left.V_{1}\left(x_{k+1}(t+1), r_{k}(t+1)\right)+V_{2}\left(y_{k+1}(t), r_{k+1}(t)\right)\right] \\
< & \mathrm{E}\left[V_{1}\left(x_{k+1}(t), r_{k}(t)\right)+V_{2}\left(y_{k}(t), r_{k}(t)\right)+\left|z_{k}(t)\right|^{2}\right. \\
& \left.+\gamma^{2}\left|w_{k}(t)\right|^{2}\right] .
\end{aligned}
$$

Moreover, evaluating (19) for $0 \leq k, t \leq N$, summing the resulting inequalities over $0 \leq k \leq N$ under the assumption that $k+t=N$ and then adding $\mathrm{E}\left[V_{1}\left(x_{N+2}(0), r_{N+1}(0)\right)+V_{2}\left(y_{0}(N+\right.\right.$ $\left.1)), r_{0}(N+1)\right]$ to both sides of the result gives

$$
\begin{aligned}
\sum_{k=0}^{N+1} \mathrm{E} & {\left[V_{1}\left(x_{k+1}(N+1-k), r_{k}(N+1-k)\right)\right.} \\
& \left.+V_{2}\left(y_{k}(N+1-k), r_{k}(N+1-k)\right)\right] \\
< & \sum_{k=0}^{N} \mathrm{E}\left[V_{1}\left(x_{k+1}(N-k), r_{k}(N-k)\right)\right. \\
& \left.+V_{2}\left(y_{k}(N-k), r_{k}(N-k)\right)\right] \\
& +\sum_{k=0}^{N} \mathrm{E}\left[\left|z_{k}(N-k)\right|^{2}-\gamma^{2}\left|w_{k}(N-k)\right|^{2}\right] .
\end{aligned}
$$

Summing both sides of this last inequality in $N$ and rearranging the resulting summands gives

$$
\begin{aligned}
\sum_{N=0}^{M} & \sum_{k=0}^{N} \mathrm{E}\left|z_{k}(N-k)\right|^{2}<\gamma^{2} \sum_{N=0}^{M} \sum_{k=0}^{N}\left|w_{k}(N-k)\right|^{2} \\
- & \sum_{k=0}^{M+1} \mathrm{E}\left[V_{1}\left(x_{k+1}(M+1-k), r_{k}(M+1-k)\right)\right. \\
& \left.+V_{2}\left(y_{k}(M+1-k), r_{k}(M+1-k)\right)\right] .
\end{aligned}
$$


Letting $M \rightarrow \infty$ gives for all $w_{k}(t) \in l_{2}([0, \infty),[0, \infty)) \neq 0$, $\|z\|_{\mathrm{E}}<\gamma\|w\|_{2}$.

One possible method of constructing the stabilizing control law $u=\varphi(\bar{x}, r)$ is to assume that the inequality

$\min _{u \in \Phi}\left\{\mathcal{D}_{\bar{x}, u, 0, r} V\left(\bar{x}_{k}(t), r_{k}(t)\right)+L(\bar{x}, u, r)\right\} \leq 0$,

has a solution $\{V(\bar{x}, r), \varphi(\bar{x}, r)\}$. Then, given Theorem 2 , the control law $u=\varphi(\bar{x}, r)$ ensures exponential stability of the controlled dynamics.

Remark 1. The results in this and the previous section have assumed time-invariant dynamics but the results obtained extend directly to time-varying dynamics.

The results in this and the previous section give existence conditions for stability and control law design and to progress to applications tractable methods of designing the control laws required must be developed. This situation mirrors that in the control theory for other classes of nonlinear systems and as in these areas further progress can be made for sub-classes where particular features that arise in modeling the dynamics can be used to advantage. The remaining part of this paper considers the ILC application where linearization about an operating point is possible, which results in time-varying models whereas the existing ILC designs in a repetitive process that have been experimentally verified require the assumption of time-invariant dynamics.

Further progress from (20) is possible for the special case of (1) described by

$$
\begin{gathered}
x_{k+1}(t+1)=A_{11}\left(r_{k}(t)\right) x_{k+1}(t)+A_{12}\left(r_{k}(t)\right) y_{k}(t) \\
+B_{1}\left(r_{k}(t)\right) u_{k+1}(t), \\
\begin{aligned}
y_{k+1}(t)= & A_{21}\left(r_{k}(t)\right) x_{k+1}(t)+A_{22}\left(r_{k}(t)\right) y_{k}(t) \\
+ & B_{2}\left(r_{k}(t)\right) u_{k+1}(t),
\end{aligned}
\end{gathered}
$$

with boundary conditions again given by (2). In particular, suppose that with $L(\bar{x}, u, r)=\bar{x}^{T} Q(r) \bar{x}+u^{T} R(r) u, \quad Q(r)=Q^{T}(r) \geq$ $0, R(r)=R^{T}(r)>0, r \in \mathbb{N}$ and $V_{1}(\bar{x}, r)=x^{T} P_{1}(r) x, V_{2}(\bar{x}, r)=$ $y^{T} P_{2}(r) y, \quad r \in \mathbb{N}$, where $\geq$ and $>$ denote the positive semidefinite and positive-definite properties. Then the control law can be obtained from

$\frac{\partial}{\partial u}\left\{\mathcal{D}_{\bar{x}, u, 0, r} V\left(\bar{x}_{k}(t), r_{k}(t)\right)+L(\bar{x}, u, r)\right\}=0$,

in the form of switching linear feedback and the matrices $P_{1}$ and $P_{2}$ obtained as a solution of a corresponding LMI problem. Similarly, in the case of stabilization and disturbance attenuation it is possible to consider the corresponding minimax problem.

In the next two sections, practically relevant applications of the general results already developed in this paper are considered. The first of these considers the case of absolute stability, which, for example, can be applied to the case when the actuator in a linear control system exhibits nonlinear dynamics. Second, the results of Theorem 1 are applied to ILC design for networks where random failures can occur.

\section{Absolute stability of a repetitive process with nonlinear actuation}

Consider the particular case of (21) when

$u_{k+1}(t)=\varphi\left(\sigma\left(i, \bar{x}_{k}(t)\right)\right) \quad$ if $r_{k}(t)=i, \varphi(0)=0$

where $\sigma\left(i, \bar{x}_{k}(t)\right)=F(i) \bar{x}_{k}(t), \quad F(i)=\left[\begin{array}{lll}F_{1}(i) & F_{2}(i)\end{array}\right], \varphi(\cdot)$ is nonlinear function, satisfying the following quadratic constraint

$\sigma^{T} Q \sigma+2 \sigma^{T} S \varphi(\sigma)+\varphi^{T}(\sigma) R \varphi(\sigma) \geq 0$, where $Q=Q^{T}, R=R^{T}$ and $S$ are matrices of appropriate dimensions. This last inequality is a standard constraint in absolute stability theory $[16,17]$. In particular, if $\sigma$ is a scalar function and $\varphi$ is a sector bounded nonlinearity, i.e.

$0 \leq \frac{\varphi(\sigma)}{\sigma} \leq k, \quad \varphi(0)=0$,

(23) has the form

$\varphi(\sigma)\left(\sigma-k^{-1} \varphi(\sigma)\right) \geq 0$.

The following definition is based on this last fact.

Definition 3. A system described by (21) is said to be absolutely stable in the class of nonlinearities (22) if it is exponentially stable in the mean square for any nonlinear function $\varphi(\sigma)$ satisfying (23).

The problem now considered is: find conditions for absolute stability of (21) in the class of nonlinearities (22) in the form suitable for efficient numerical verification.

Choose the components of the vector Lyapunov function (6) in this case as the quadratic forms

$V_{1}=x_{k+1}^{T}(t) P_{1}\left(r_{k}(t)\right) x_{k+1}(t), \quad V_{2}=y_{k}^{T}(t) P_{2}\left(r_{k}(t)\right) y_{k}(t)$,

To ensure the absolute stability of the system (21) with the control law (22) applied, this particular case of the function (6) must satisfy the conditions of Theorem 1 for all $\varphi(\sigma)$ satisfying (23). Applying the $S$-procedure [17] gives that these conditions hold and (21) is absolute stable if $P(i)=P_{1}(i) \oplus P_{2}(i)>0, i \in \mathbb{N}$ and for sufficiently small arbitrary $\varepsilon>0$

$$
\begin{aligned}
& {\left[\begin{array}{cc}
A^{T}(i) H(i) A(i)-P(i)+F^{T}(i) Q F(i)+\varepsilon I & A^{T}(i) H(i) B(i)+F^{T}(i) S \\
B^{T}(i) H(i) A(i)+S^{T} F(i) & R+B^{T}(i) H(i) B(i)
\end{array}\right]} \\
& \leq 0, \quad i \in \mathbb{N},
\end{aligned}
$$

where $A(i)=\left[\begin{array}{ll}A_{11}(i) & A_{12}(i) \\ A_{21}(i) & A_{22}(i)\end{array}\right], \quad H(i)=\sum_{j=1}^{v} \pi_{i j} P_{1}(j) \oplus \sum_{j=1}^{v} \omega_{i j}$ $P_{1}(j), B(i)=\left[\begin{array}{l}B_{1}(i) \\ B_{2}(i)\end{array}\right], \quad i \in \mathbb{N}$ and $\oplus$ denotes the direct sum of two matrices. Also the inequalities (25) are easily checked using standard LMI software.

\section{ILC application}

As briefly discussed in the Introduction section of this paper, ILC is applicable to systems that repeat the same finite duration task over and over again. In ILC design, the input on pass $k+1$ is a function of the input on the previous term and a correction term involving the error on the previous pass $k$, i.e., of the form $u_{k+1}=h\left(u_{k}, \Delta_{k}\right)$ where $\Delta_{k}$ denotes the this term.

As one application of the stability theory developed in the previous section, ILC design over a leader-follower network is considered where the dynamics of the subsystems linearized about an operating point are represented by time-varying linear models with uncertainty. The ILC design algorithms that have seen experimental verification, such as those in [4], assume that the system dynamics are time-invariant or can be well approximated by such a model. Research has also been reported on linear and nonlinear model based ILC for time-varying dynamics, such as [18], but there is no one way of addressing this issue and it is only when a number of methods have been investigated in significant depth that will it be possible to do comparative studies. The analysis that follows allows failures to be included and extends to LMIs for design.

The leader-follower network considered consists of $N$ systems, each of which is described a state-space model of the form

$x^{n}(t+1)=A^{(n)}(\delta(t)) x^{n}(t)+B^{(n)}(\delta(t)) u^{n}(t)$,

$y^{n}(t)=C^{(n)} x^{n}(t), \quad n=1,2, \ldots, N, 0 \leq t \leq T$, 
where for the $n$th system $x^{n}(t)$ is the state vector, $u^{n}(t)$ is the input vector, $y^{n}$ is the output vector, $\delta(t)$ is the vector of uncertain parameters and $A^{(n)}, B^{(n)}$ and $C^{(n)}$ denote the model matrices. Also the dynamics have associated uncertainty that is assumed to be of the affine parallelotopic type with variations around a central nominal model defined by the matrices $\left(A^{(n)}, B^{(n)}\right)$ along the axes $\left(A^{(n)_{l}}, B^{(n)_{l}}\right)$ of the form

$$
\begin{aligned}
& A^{(n)}(\delta(t))=A^{(n)}+\sum_{l=1}^{M} \delta_{l}(t) A^{(n)_{l},}, \\
& B^{(n)}(\delta(t))=B^{(n)}+\sum_{l=1}^{M} \delta_{l}(t) B^{(n)_{l},},
\end{aligned}
$$

where $M$ denotes the number of uncertain parameters and the uncertainty region $\Delta$ is defined as

$$
\begin{aligned}
\boldsymbol{\Delta}= & \left\{\delta(t)=\left[\delta_{1}(t) \ldots \delta_{M}(t)\right]^{T}: \underline{\delta}_{l} \leq \delta_{l} \leq \bar{\delta}_{l}, \underline{\delta}_{l} \leq 0,\right. \\
& \left.\bar{\delta}_{l} \geq 0, \quad l=1, \ldots, M\right\} .
\end{aligned}
$$

Define also the finite set

$\boldsymbol{\Delta}_{v}=\left\{\delta=\left[\delta_{1}, \ldots, \delta_{M}\right]^{T}: \delta_{l} \in\left\{\underline{\delta}_{l}, \bar{\delta}_{l}\right\}, l=1, \ldots, M\right\}$,

representing the vertices of the convex hull in which the uncertain parameters take their values. Again the subscript $k$ is added to the vector variables to denote the ILC pass number.

In this paper it is assumed that only one system, termed the leader, tracks the reference signal directly and the others track the leader. If a follower loses communication from the leader due to insufficient reliability of the network, it will track the available follower and the system will track the leader again when access to the leader is restored. It is assumed that at each time instant only one of the followers can be out of contact with the leader. Network failures are also considered where these are assumed to be modeled by the Markov chain $r(t)$ with finite set of states $\mathbb{N}=\{1, \ldots, v\}$ and transition probabilities given by

$\mathrm{P}[r(t+1)=j \mid r(t)=i]=\pi_{i j}$.

Suppose that $y_{\text {ref }}(t)$ denotes the reference vector. Then on pass $k$, the errors of the leader and followers are given by

$e_{k}^{1}(t)=y_{\text {ref }}(t)-y_{k}^{1}(t)$,

$e_{k}^{n}(t)=y_{k}^{I_{n}[r(t)]}-y_{k}^{n}(t), \quad n=2, \ldots, N$,

where $I_{n}[r(t)]$ can take values from 1 to $N$, except $n$, depending on the failures in the network, i.e., on the state of the Markov chain $r(t)$. Also it is assumed that the controlled system starts each pass from a state vector with constant entries. The problem is to design an ILC law such that

$$
\lim _{k \rightarrow \infty} E\left[\left|e_{k}^{n}(t)\right|\right]=0, \quad \lim _{k \rightarrow \infty} E\left[\left|u_{k}^{n}(t)-u_{\infty}^{n}(t)\right|\right]=0,
$$

A common form of ILC law for linear dynamics is to select the input on the current pass as that used on the previous one plus a correction term, i.e.,

$u_{k+1}^{n}(t)=u_{k}^{n}(t)+\Delta u_{k+1}^{n}(t)$,

where $\Delta u_{k+1}^{n}(t)$ denotes the correction used to update the control input on the previous pass for use on the next pass, where once a pass is complete all information generated during its evolution is available for use in constructing this term.

Introduce, for analysis purposes only,

$\xi_{k+1}^{n}(t+1)=x_{k+1}^{n}(t)-x_{k}^{n}(t), \quad n=1, \ldots, N$,

$\xi_{k}(t)=\left[\left(\xi_{k}^{1}\right)^{T}(t) \ldots\left(\xi_{k}^{N}\right)^{T}(t)\right]^{T}$,

$e_{k}=\left[\left(e_{k}^{1}\right)^{T}(t) \ldots\left(e_{k}^{N}\right)^{T}(t)\right]^{T}$.
Suppose also that the state vector $x_{k}(t)$ is available for use and consider the following form of the correction term in the control law (33):

$\Delta u_{k+1}(t)=F_{1}(i) \xi_{k+1}(t+1)+F_{2}(i) e_{k}(t+1)$,

if $r(t)=i, i \in \mathbb{N}$,

where $\Delta u_{k}(t)=\left[\Delta u_{k}^{1}(t)^{T} \ldots \Delta u_{k}^{n}(t)^{T}\right]^{T}$ and

$F_{1}(i)=\left[\begin{array}{cccc}F_{1}^{(1)}(i) & 0 & \ldots & 0 \\ 0 & F_{1}^{(2)}(i) & \ldots & 0 \\ 0 & 0 & \ldots & F_{1}^{(N)}(i)\end{array}\right]$,

$F_{2}(i)=\left[\begin{array}{cccc}F_{2}^{(1)}(i) & 0 & \ldots & 0 \\ 0 & F_{2}^{(2)}(i) & \ldots & 0 \\ 0 & 0 & \ldots & F_{2}^{(N)}(i)\end{array}\right]$.

Using (34), the ILC law for this application can be written as

$u_{k+1}(t)=u_{k}(t)+F_{1}(i)\left[x_{k+1}(t)-x_{k}(t)\right]+F_{2}(i) e_{k}(t+1)$,

if $r(t)=i, i \in \mathbb{N}$,

where the term $e_{k}(t+1)$ is causal and hence implementable since it is generated on the previous pass. The ability to use non-causal temporal information in control law design is the novel property of ILC.

The ILC dynamics can be written as

$$
\begin{aligned}
\xi_{k+1}(t+1)= & {\left[A_{11}(\delta(t))+B_{1}(\delta(t)) F_{1}(r(t-1))\right] \xi_{k+1}(t) } \\
& +B_{1}(\delta(t)) F_{2}(r(t-1)) e_{k}(t), \\
e_{k+1}(t)= & {\left[A_{21}(\delta(t), r(t))+B_{2}(\delta(t), r(t)) F_{1}(r(t-1))\right] \xi_{k+1}(t) } \\
+ & {\left[I+B_{2}(\delta(t), r(t)) F_{2}(r(t-1))\right] e_{k}(t), }
\end{aligned}
$$

where $A_{11}=\operatorname{diag}\left[A^{(1)}, \ldots, A^{(N)}\right]$,

$B_{1}=\left[\begin{array}{cccc}B^{(1)} & 0 & \cdots & 0 \\ 0 & B^{(2)} & \cdots & 0 \\ \vdots & \vdots & \ddots & \vdots \\ 0 & 0 & \cdots & B^{(N)}\end{array}\right]$,

and the matrices $A_{21}$ and $B_{2}$ have a random structure determined by the failures that occur. In the absence of failures, each follower receives information from the leader (system 1 ) and these matrices have the following form

$A_{21}=\left[\begin{array}{ccccc}-C^{(1)}(i) A^{(1)}(\delta) & 0 & 0 & \ldots & 0 \\ C^{(1)}(i) A^{(1)}(\delta) & -C^{(2)}(i) A^{(2)}(\delta) & 0 & \ldots & 0 \\ \vdots & \vdots & \vdots & \ldots & \vdots \\ C^{(1)}(i) A^{(1)}(\delta) & 0 & \ldots & 0 & -C^{(N)}(i) A^{(N)}(\delta)\end{array}\right]$

$B_{2}=\left[\begin{array}{ccccc}-C^{(1)}(i) B^{(1)}(\delta) & 0 & 0 & \ldots & 0 \\ C^{(1)}(i) B^{(1)}(\delta) & -C^{(2)}(i) B^{(2)}(\delta) & 0 & \ldots & 0 \\ \vdots & \vdots & \vdots & \ldots & \vdots \\ C^{(1)}(i) B^{(1)}(\delta) & 0 & \ldots & 0 & -C^{(N)}(i) B^{(N)}(\delta)\end{array}\right]$.

When failures occur in system $n$, the entries $-C^{(n)} A^{(n)}$ and $-C^{(n)} B^{(n)}$ in the above matrices remain in place but the other entries can change. By way of example, consider the case when $N=3$, where if the first follower (system 2) loses communication with the leader then

$A_{21}=\left[\begin{array}{ccc}-C^{(1)} A^{(1)} & 0 & 0 \\ 0 & -C^{(2)} A^{(2)} & C^{(3)} A^{(3)} \\ C^{(1)} A^{(1)} & 0 & -C^{(3)} A^{(3)}\end{array}\right]$,

$B_{2}=\left[\begin{array}{ccc}-C^{(1)} B^{(1)} & 0 & 0 \\ 0 & -C^{(2)} B^{(2)} & C^{(3)} B^{(3)} \\ C^{(1)} B^{(1)} & 0 & -C^{(3)} B^{(3)}\end{array}\right]$. 
If the second follower (system 3) loses communication with the leader then

$A_{21}=\left[\begin{array}{ccc}-C^{(1)} A^{(1)} & 0 & 0 \\ C^{(1)} A^{(1)} & -C^{(2)} A^{(2)} & 0 \\ 0 & C^{(2)} A^{(2)} & -C^{(3)} A^{(3)}\end{array}\right]$,
$B_{2}=\left[\begin{array}{ccc}-C^{(1)} B^{(1)} & 0 & 0 \\ -C^{(1)} B^{(1)} & -C^{(2)} B^{(2)} & 0 \\ 0 & C^{(2)} B^{(2)} & -C^{(3)} B^{(3)}\end{array}\right]$,

(the dependence on $\delta$ and $i$ is omitted for ease of notation).

It is routine to establish that pass-to-pass error convergence will occur if exponential stability holds. To obtain conditions for this property, consider a vector Lyapunov function of the form (6) with

$V_{1}=\xi_{k+1}^{T}(t) P_{1}(r(t)) \xi_{k+1}(t)$,

$P_{1}(i)=\operatorname{diag}\left[P_{1}^{(1)}(i), \ldots, P_{1}^{(N)}(i)\right]$,

$V_{2}=e_{k}^{T}(t) P_{2}(r(t)) e_{k}(t)$,

$P_{2}(i)=\operatorname{diag}\left[P_{2}^{(1)}(i), \ldots, P_{2}^{(N)}(i)\right]$.

By Theorem 2 the controlled system is exponentially stable when

$P(i)>0$,

$\sum_{j=1}^{\nu} \bar{A}_{c i}^{T}(\delta, j) \bar{P}_{i}(j) \bar{A}_{c i}(\delta, j)-P(i)+\bar{Q}(i) \leq 0, \quad i \in \mathbb{N}, \delta \in \Delta$,

where

$\bar{A}_{c i}(\delta, j)=\left[\begin{array}{cc}A_{11}(\delta)+B_{1}(\delta) F_{1}(i) & A_{12}(\delta)+B_{1}(\delta) F_{2}(i) \\ A_{21}(\delta, j)+B_{2}(\delta, j) F_{1}(i) & I+B_{2}(\delta, j) F_{2}(i)\end{array}\right]$,

$P(i)=\operatorname{diag}\left[P_{1}(i), P_{2}(i)\right], \quad \bar{P}_{i}(j)=\operatorname{diag}\left[P_{1}(j) P_{2}(i)\right]$,

$\bar{Q}(i)=Q(i)+R(i), \quad Q(i)=\operatorname{diag}\left[Q_{1}(i), Q_{2}(i)\right]$,

$R(i)=\operatorname{diag}\left[F_{1}^{T}(i) R_{1}(i) F_{1}(i), F_{2}^{T}(i) R_{2}(i) F_{2}(i)\right]$.

Using the Schur's complement formula, the stability conditions (37) can be reduced to the following finite set of LMIs

$\left[\begin{array}{ccc}M_{11}(i) & M_{12}(\delta, i) & M_{13}(i) \\ M_{12}(\delta, i)^{T} & M_{22}(i) & 0 \\ M_{13}(i)^{T} & 0 & M_{33}(i)\end{array}\right] \geq 0$

$X(i)=\operatorname{diag}\left[X_{1}(i), \quad X_{2}(i)\right]>0, \quad i=1, \ldots, \mathbb{N}, \quad \delta \in \Delta_{v}$,

where

$X_{1}(i)=\operatorname{diag}\left[X_{1}^{(1)}(i), \ldots, X_{1}^{(N)}(i)\right]$,

$X_{2}(i)=\operatorname{diag}\left[X_{2}^{(1)}(i), \ldots, X_{2}^{(N)}(i)\right], \quad M_{11}(i)=X(i)$,

$M_{12}(\delta, i)=\left[M_{121}(\delta, i) M_{122}(\delta, i) \ldots M_{12 v}(\delta, i)\right]$,

$M_{12 j}(\delta, i)=\pi_{i j}^{1 / 2}$

$\times\left[\begin{array}{cc}\left(A_{11}(\delta) X_{1}(i)+B_{1}(\delta) Y_{1}(i)\right) & B_{1}(\delta) Y_{2}(i) \\ \left(A_{21}(\delta, j) X_{1}(i)+B_{2}(\delta, i) Y_{1}(i)\right) & X_{2}(i)+B_{2}(\delta, j) Y_{2}(i)\end{array}\right]^{T}$,

$M_{13}(i)=\left[X(i) Y^{T}(i)\right], Y(i)=\left[Y_{1}(i) Y_{2}(i)\right]$,

$Y_{1}(i)=F_{1}(i) X_{1}(i)$

$Y_{2}(i)=F_{2}(i) Y_{2}(i), \quad M_{33}(i)=\operatorname{diag}\left[Q^{-1}(i), R^{-1}(i)\right]$,

$M_{22}(i)=\operatorname{diag}\left[X_{1}(1) X_{2}(i), X_{1}(2) X_{2}(i), \ldots, X_{1}(v) X_{2}(i)\right]$,

and the following result has been established.
Theorem 4. Suppose that the LMIs given as (38) are feasible with respect to the variables

$X_{1}(i)=\operatorname{diag}\left[X_{1}^{(1)}(i), \ldots, X_{1}^{(N)}(i)\right]$,

$X_{2}(i)=\operatorname{diag}\left[X_{2}^{(1)}(i), \ldots, X_{2}^{(N)}(i)\right]$,

$Y_{1}(i)=\left[\begin{array}{cccc}Y_{1}^{1}(i) & 0 & \cdots & 0 \\ 0 & Y_{1}^{2}(i) & \cdots & 0 \\ 0 & 0 & \cdots & Y_{1}^{N}(i)\end{array}\right]$

$Y_{2}(i)=\left[\begin{array}{cccc}Y_{2}^{1}(i) & 0 & \cdots & 0 \\ 0 & Y_{2}^{2}(i) & \cdots & 0 \\ 0 & 0 & \cdots & Y_{2}^{N}(i)\end{array}\right]$

Then a networked system descried by (26)-(29) operating under an ILC law of the form (33) with correction term defined by (35) is convergent with

$F_{1}(i)=Y_{1}(i) X_{1}^{-1}(i), \quad F_{2}(i)=Y_{2}(i) X_{2}^{-1}(i), \quad i=1, \ldots, \mathbb{N}$.

Often the transition probabilities of the Markov chain $r(t)$ are unknown and one option in this case is to find a conservative solution in the form of a non-switching feedback control law obtained using a common Lyapunov function. This function has the form (6) with

$V_{1}=\xi_{k+1}^{T}(t) P_{1} \xi_{k+1}(t), \quad P_{1}=\operatorname{diag}\left[P_{1}^{(1)}, \ldots, P_{1}^{(N)}\right]$,

$V_{2}=e_{k}^{T}(t) P_{2} e_{k}(t)$,

$P_{2}=\operatorname{diag}\left[P_{2}^{(1)}, \ldots, P_{2}^{(N)}\right]$,

i.e., the matrices $P_{1}$ and $P_{2}$ are independent of $r(t)$. In this case, the following corollary to the last theorem holds.

Corollary 2. Suppose that LMIs

$\left[\begin{array}{cccc}X & \bar{A}_{c}^{T}(\delta, i)^{T} & X & Y^{T} \\ A_{c}(\delta, i) & X & 0 & 0 \\ X & 0 & Q^{-1} & 0 \\ Y & 0 & 0 & R^{-1}\end{array}\right] \geq 0$

$i=1, \ldots, N, \delta \in \Delta_{v}$,

are feasible with respect to $X$ and $Y$, which are the counterparts of those in the previous theorem but with constant entries. Then a networked system descried by (26)-(29) with an ILC law of the form (33) and (35) applied is convergent when $F_{1}$ and $F_{2}$ are given by

$F_{1}=Y_{1} X_{1}^{-1}, \quad F_{2}=Y_{2} X_{2}^{-1}$.

\subsection{Numerical example}

Consider the case of (26) with $N=3$. Suppose also that the leader has no uncertainty associated with its dynamics and its state-space model matrices are

$A^{(1)}=\left[\begin{array}{ccc}-0.002961 & 1 & 0 \\ -0.0008363 & -0.002961 & 0.3035 \\ 0 & 0 & 0\end{array}\right]$,

$B^{(1)}=\left[\begin{array}{lll}0 & 0 & 0.1563\end{array}\right]^{T}$,

$C^{(1)}=\left[\begin{array}{lll}0.0003718 & 0.007077 & 0.02335\end{array}\right]$

where the entries in the matrices of $A^{(n)}$ and $B^{(n)}(n=2,3)$ of the state-space models for the followers have variations up to $20 \%$ of those in the leader model matrices given above. Suppose also that the possible failures are modeled as an homogeneous Markov 


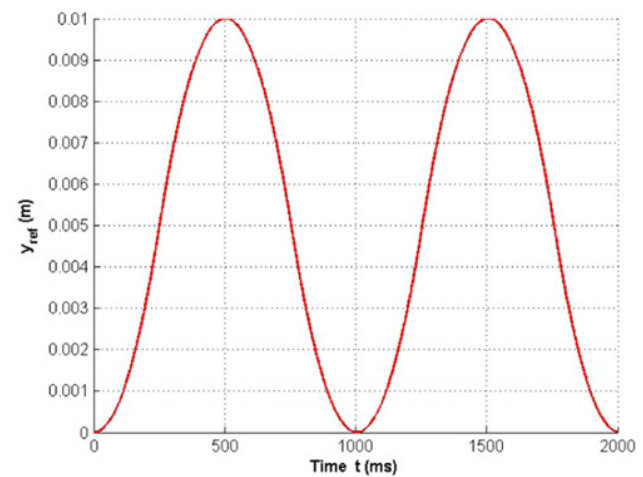

Fig. 1. Reference signal.

process with three states corresponding to three possible modes. In the first mode, the normal configuration, both the followers track the leader. In the second, failure 1, the first follower loses information from the leader and tracks the second follower and in the third, failure 2, the second follower loses information from the leader and tracks the first follower.

In the case when the transition probabilities between these states are unknown, the non-switching feedback control law is obtained from Corollary 2. Solving the LMIs of this result and using (40) gives

$F_{1}^{(1)}=F_{1}^{(2)}=F_{1}^{(3)}=[-0.0096-0.2814-51.9978]$,

$F_{2}^{(1)}=F_{2}^{(2)}=F_{1}^{(3)}=922.88$.

The reference signal for this example is shown in Fig. 1. If the network has no failures then the error for each system decreases monotonically from pass-to-pass ( $k$ ), see Fig. 2. Fig. 3 shows the simulation results for the following scenario: on pass 5 the first follower loses contact with the leader and switches to tracking the second follower, from pass 7 the leader is available again and the first follower continues to track the leader, on pass 10 the second follower loses the leader and switches to tracking the first follower, on pass 15 the leader is available again and the second follower

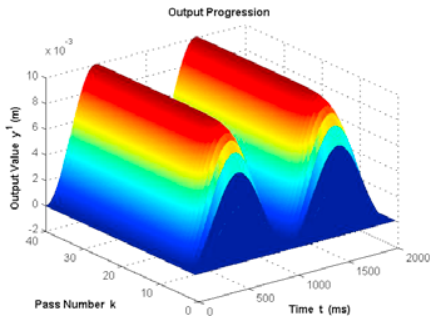

(a) Output of the leader.

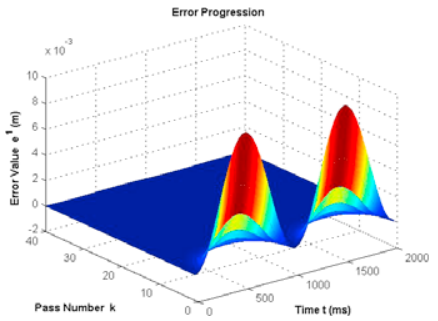

(b) Error of the leader.

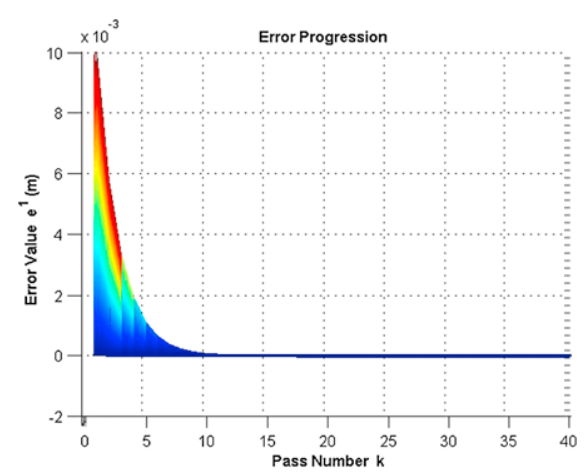

(c) Error of the leader (side view).

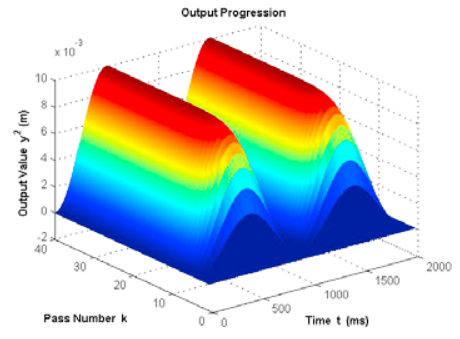

(d) Output of the 1-st follower.

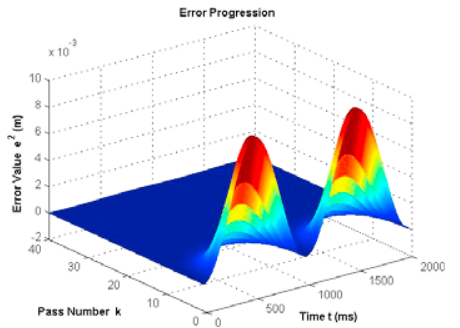

(e) Error of the 1-st follower.

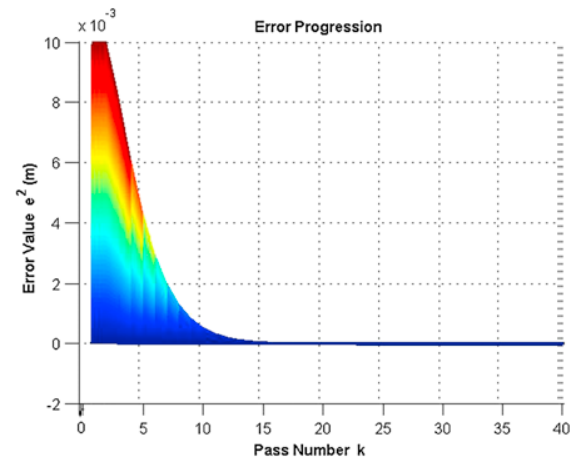

(f) Error of the 1-st follower (side view).

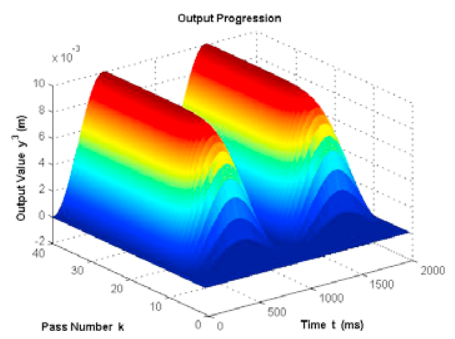

(g) Output of the 2-nd follower.

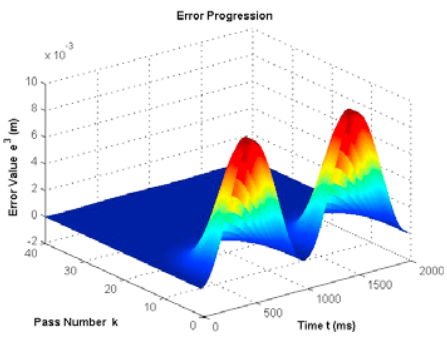

(h) Error of the 2-nd follower.

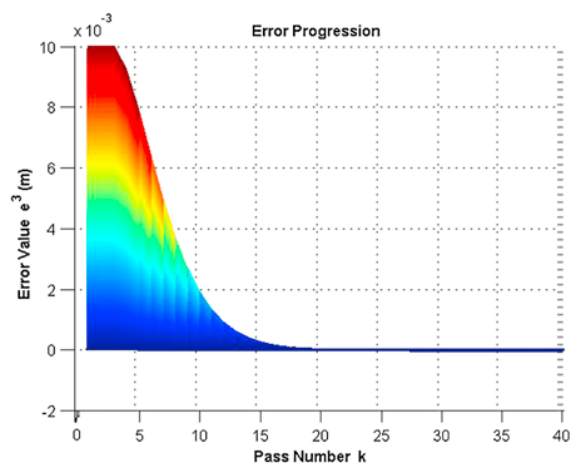

(i) Error of the 2-nd follower (side view).

Fig. 2. Simulation results for the network with no failures. 


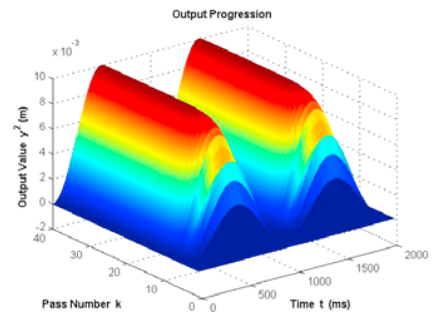

(a) Output of the 1-st follower (loss of the leader on the 5 -th pass).

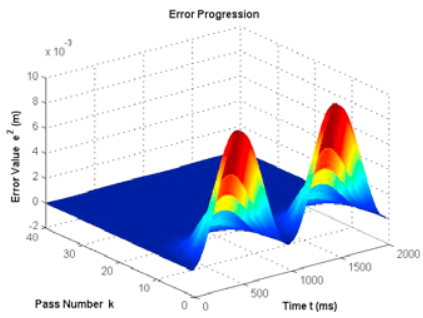

(b) Error of the 1-st follower (loss of the leader on the 5 -th pass).

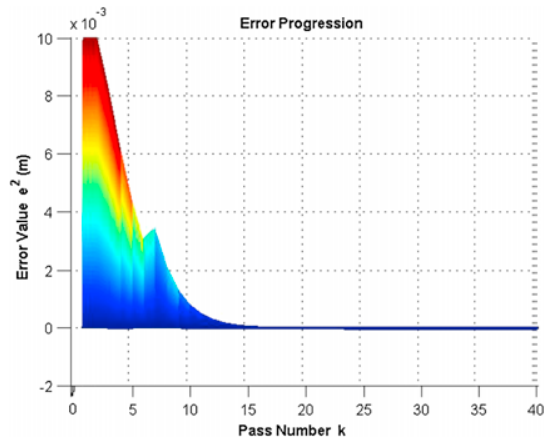

(c) Error of the 1-st follower (loss of the leader on the 5-th pass, side view).

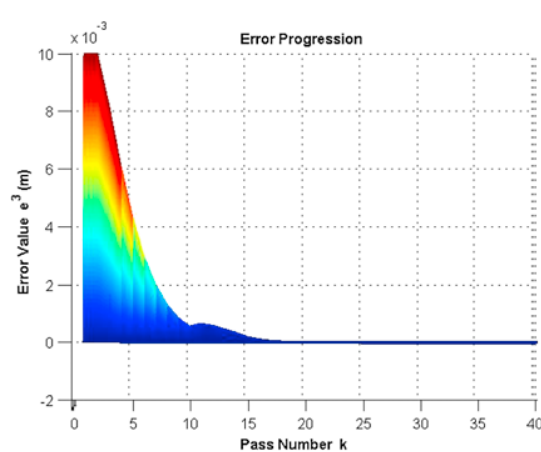

(f) Error of the 2-nd follower (loss of the leader on the 10 -th pass, side view).

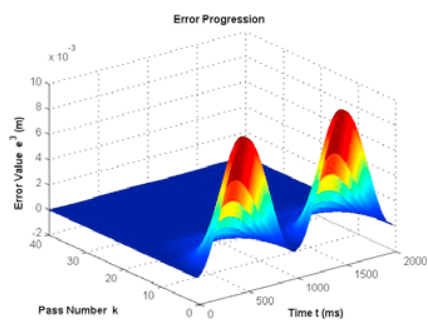

(e) Error of the 2-nd follower (loss of the leader on the 10-th pass).

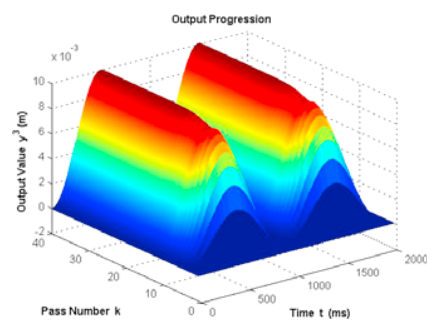

the leader on the 10-th pass)

Fig. 3. Simulation results for the network with failures.

continues to track the leader. In the presence of failures, the error convergence from pass-to-pass can be non-monotonic.

\section{Conclusions}

This paper has developed a stability theory that is applicable to discrete nonlinear time-varying repetitive processes. The analysis extends to conditions for stability under control action with disturbance attenuation as measured by an $\mathscr{H}_{\infty}$ norm and the presence of failures as described by Markovian switching. In common with other control theory for nonlinear dynamics, progress from these results towards application will be enabled by exploiting any extra structural properties of the dynamics in a given application area. As one example of this latter point, the application of the new theory to ILC law design has been considered where the results obtained add to an area that is still in the relatively early stages of development. The results obtained allow nonlinear time-varying dynamics in both the nominal model and that for the uncertainty structure assumed. Current research is aimed at fully exploiting the potential of basing ILC design on time-varying approximation of the dynamics about an operating point. Progress in this respect will also widen the applications for 2D nonlinear control theory.

\section{References}

[1] E. Rogers, K. Gałkowski, D.H. Owens, Control Systems Theory and Applications for Linear Repetitive Processes, in: Lecture Notes in Control and Information Sciences, vol. 349, Springer-Verlag, Berlin, Germany, 2007.

[2] D.A. Bristow, M. Tharayil, A. Alleyne, A survey of iterative learning control, IEEE Control Syst. Mag. 26 (3) (2006) 96-114.

[3] H.-S. Ahn, Y.-Q. Chen, K.L. Moore, Iterative learning control: Brief survey and categorization, IEEE Trans. Syst. Man Cybern. C 37 (6) (2007) 1099-1121.
[4] L. Hladowski, K. Galkowski, Z. Cai, E. Rogers, C.T. Freeman, P.L. Lewin, Experimentally supported $2 D$ systems based iterative learning control law design for error convergence and performance, Control Eng. Practice 18 (4) (2010) 339-348.

[5] Nima Yeganefar, Nader Yeganefar, M. Ghamgui, E. Moulay, Lyapunov theory for 2-D nonlinear Roesser models: Application to asymptotic and exponential stability, IEEE Trans. Automat. Control 58 (2013) 1299-1304.

[6] P.M. Sammons, D.A. Bristow, R.G. Landers, Iterative learning control of bead morphology in laser metal deposition processes, in: Proc. American Control Conference, Washington, DC, June 17-19, 2013, pp. 5962-5967.

[7] O.L.V. Costa, M.D. Fragoso, R.P. Marques, Discrete-Time Markov Jump Linear Systems, Springer, New York, 2004.

[8] J. Baczynski, M.D. Fragoso, Maximal versus strong solution to algebraic Riccati equations arising in infinite Markov jump linear systems, Systems Control Lett. 57 (2008) 246-254

[9] R.C.L.F. Oliveira, A.N. Vargas, J.B.R. do Val, P.L.D. Peres, Robust stability, $H_{2}$ analysis and stabilisation of discrete-time Markov jump linear systems with uncertain probability matrix, Internat. J. Control 82 (2009) 470-481.

[10] P. Pakshin, D. Peaucelle, LQR parametrization of static output feedback gains for linear systems with Markovian switching and related robust stabilization and passification problems, in: Proceedings of the Joint 48th IEEE Conference on Decision and Control and 28th Chinese Control Conference, Shanghai, China, December 16-18, 2009, pp. 1157-1162.

[11] W. Li, H. Song, Y. Qu, K. Wang, Global exponential stability for stochastic coupled systems on networks with Markovian switching, Systems Control Lett. 62 (2013) 468-474

[12] C. Li, M.Z.Q. Chen, J. Lam, X. Mao, On exponential almost sure stability of random jump systems, IEEE Trans. Automat. Control 57 (2012) 3064-3077.

[13] O.L.V. Costa, D.Z. Figueiredo, Stochastic stability of jump discrete-time linear systems with Markov chain in a general Borel space, IEEE Trans. Automat. Control 57 (2014) 223-227.

[14] H. Gao, J. Lam, S. Xu, C. Wang, Stabilization and $H_{\infty}$ control of two-dimensional Markovian jump systems, IMA J. Math. Control Inf. 21 (2004) 377-392.

[15] L. Wu, P. Shi, H. Gao, C. Wang, $H_{\infty}$ filtering for 2D Markovian jump systems, Automatica 44 (2008) 1849-1858.

[16] M. Vidyasagar, Nonlinear Systems Analysis, SIAM, Philadelphia, 2002.

[17] S. Boyd, L. El-Ghaoui, E. Feron, V. Balakrishnan, Linear Matrix Inequalities in System and Control Theory, SIAM, Philadelphia, 1994.

[18] W. Yan, M. Sun, Adaptive iterative learning control of discrete-time varying systems with unknown control direction, Internat. J. Adapt. Control Signal Process. 27 (2013) 340-348. 